\section{Relación entre prácticas pedagógicas y estrategias de aprendizaje en docentes de carreras de la salud}

\author{
CONSTANZA CARRASCO Z. ${ }^{1, a}$, CRISTHIAN PÉREZ V., \\ GRACIELA TORRES A. ${ }^{1, c}$, EDUARDO FASCE H. ${ }^{2}$
}

\section{Teaching practices and learning strategies in health careers}

\begin{abstract}
Background: Medical Education, according to the constructivist education paradigm, puts students as the protagonists of the teaching and learning process. It demands changes in the practice of teaching. However, it is unclear whether this new model is coherent with the teachers' ways to cope with learning. Aim: To analyze the relationship between teaching practices and learning strategies among teachers of health careers in Chilean universities. Material and Methods: The Teaching Practices Questionnaire and Learning Strategies Inventory of Schmeck were applied to 200 teachers aged 24 to 72 years ( $64 \%$ females). Results: Teachers use different types of teaching practices. They commonly use deep and elaborative learning strategies. A multiple regression analysis showed that learning strategies had a $13 \%$ predictive value to identify student-centered teaching, but they failed to predict teacher-centered teaching. Conclusions: Teaching practices and learning strategies of teachers are related. Teachers frequently select constructivist model strategies, using different teaching practices in their work
\end{abstract}

(Rev Med Chile 2016; 144: 1199-1206)

Key words: Education, Medical, Undergraduate; Faculty, Medical; Learning.
'Dirección de la Carrera de Medicina. Facultad de Medicina, Universidad San Sebastián. Concepción, Chile.

${ }^{2}$ Departamento de Educación Médica, Facultad de Medicina, Universidad de Concepción.

Concepción, Chile.

aMagíster en Educación Médica para las Ciencias de la Salud.

besicólogo, Magíster en Psicología con mención en Psicología Educativa.

'Enfermera-Matrona, Magíster en Educación Médica para las Ciencias de la Salud.

Recibido el 24 de agosto de 2015, aceptado el 2 de junio de 2016.

Correspondencia a:

Constanza Carrasco Zunino

Facultad de Medicina Tercer Piso Edificio Los Robles,

Campus Tres Pascualas, Universidad San Sebastián. Cruz № 1577, Concepción Chile. constanza.carrasco@uss.cl

\section{A} tendiendo a los cambios sociales, la educación en carreras de la salud está fomentando la creación de programas que se adapten al rápido y permanente avance de la ciencia y la tecnología $a^{1-3}$.

Responder a estos cambios implica, principalmente, posicionar al estudiante como el centro del proceso de enseñanza-aprendizaje y al docente como el facilitador ${ }^{4}$. De esta forma, el docente debe adoptar nuevas competencias que le permitan facilitar efectivamente los aprendizajes y asegurar el desarrollo de competencias profesionales ${ }^{5,6}$.

Actualmente en Chile, en las carreras de la salud, distintos graduados del área pueden desempeñarse como docentes, aun careciendo de competencias pedagógicas. Esto lleva a que, habiéndose formado en sistemas tradicionales, tiendan a repetir los modelos experimentados, centrado en el profesor ${ }^{7}$.

Por esto, se ha despertado un gran interés en cómo los docentes realizan su labor, esto es, sus prácticas pedagógicas, las que conceptualmente corresponden a los métodos de enseñanza o al conjunto de momentos y técnicas lógicamente ordenados para dirigir el aprendizaje del estudiante hacia determinados objetivos ${ }^{8}$. Éstas son afectadas por los significados y percepciones del docente, las acciones de todos los involucrados en el proceso educativo, y los aspectos político-institucionales, administrativos y normativos, que según el proyecto educativo de cada país, delimitan el rol del maestro 9 .

Así, es esperable que -además de lo ya mencionado- las prácticas pedagógicas de un docente estén asociadas a la forma en que éste concibe el aprendizaje. De hecho, según Valcárcel y Verdú ${ }^{10}$, 
mejorar la enseñanza requiere una comprensión más clara y exhaustiva del proceso de aprender.

Pero, ¿cómo identificar la forma en que las personas aprenden? Al respecto, existen dos conceptos de uso habitual: estilos y estrategias de aprendizaje.

Para Schmeck, el estilo de aprendizaje es el estilo cognitivo que un individuo manifiesta al enfrentar una tarea de aprendizaje. Las estrategias de aprendizaje, en cambio, son elecciones conscientes, entre diversas alternativas, de procedimientos para aprender, seleccionadas en función de los requerimientos de cada tarea ${ }^{10}$.

Schmeck identifica cuatro estrategias de aprendizaje, que a diferencia de otros modelos, busca diferenciar el grado de profundidad de procesamiento de la información que se alcanza al aprender, según la estimulación y los requerimientos ambientales ${ }^{11}$ :

1. Procesamiento profundo: donde el aprendiz basa su aprendizaje en las asociaciones que le sugiere el concepto, es decir, pone atención a los rasgos semánticos y es capaz de clasificar, comparar, contrastar, analizar y sintetizar la información ${ }^{11}$.

2. Procesamiento elaborativo: donde la información se vuelve personalmente más relevante. Así, el aprendiz la elabora pensando en ejemplos personales y logra expresarla en sus propias palabras. Implica adaptación, aplicación y desarrollo de mecanismos de almacenamiento, lo que acarrea un aumento en la complejidad y riqueza del aprendizaje ${ }^{11}$.

3. Estudio metódico: tiene relación con distribución y organización del tiempo de estudio. Implica aplicar meticulosamente las técnicas de estudio y se basa en la lectura reiterativa de la información tal cual es presentada hasta memorizarla ${ }^{1}$.

4. Retención de hechos: no es otra estrategia independiente, sino el extremo inferior en el continuo del procesamiento profundo. El aprendiz que utiliza esta estrategia, preferentemente asimila la información mecánicamente, tal como la recibe ${ }^{11}$.

El presente estudio parte de la premisa que una docencia que responda a las nuevas demandas educativas, logrando ejercerse con foco en el estudiante y en el desarrollo de competencias profesionales ${ }^{4-6}$, requiere de académicos con procesos de aprendizaje y concepciones sobre el mismo, que sean coherentes con el nuevo modelo que se les demanda.

No obstante, no existe evidencia en carreras de la salud sobre la relación entre las formas en que el docente enseña y aprende, por lo que el presente estudio se plantea como objetivos: caracterizar las prácticas pedagógicas y las estrategias de aprendizaje de docentes de carreras de la zona centro-sur de Chile, para, posteriormente, evaluar si existe relación entre ellas.

\section{Método}

Se realizó un estudio cuantitativo y analítico-relacional, aplicando un diseño no experimental y transversal.

\section{Participantes}

La población del estudio estuvo compuesta de profesionales con al menos un año de experiencia docente en carreras de la salud de universidades acreditadas de la zona centro-sur de Chile. Se seleccionó 200 docentes mediante muestreo no probabilístico por accesibilidad, siendo 128 (64,0\%) mujeres y $72(36,0 \%)$ hombres, con edades entre 24 y 72 años $(M=38,47 ; \mathrm{DE}=10,23)$.

Sesenta y dos por ciento $(n=124)$ tenía estudios de magíster, con 66 de ellos $(33,00 \%)$ ya graduados y 33 (16,50\%) en etapa de tesis. De éstos, 63 $(31,50 \%)$ cursaban un programa de magíster en temáticas relacionadas con la educación (educación médica, docencia universitaria, pedagogía, etc.). Sólo 15 (7,50\%) tenían estudios doctorales. Todos realizaban docencia de pregrado, con una experiencia de 1 a 41 años $(M=9,41 ; D E=7,86)$. De éstos, 93 (46,50\%) reportó hacerlo en universidades tradicionales y $153(75,50 \%)$ en privadas, con $51(25,50 \%)$ trabajando en ambos tipos de entidades.

\section{Instrumentos}

Cuestionario de Prácticas Pedagógicas (CCP): Presenta 60 ítems que abordan prácticas pedagógicas de enseñanza, evaluación, planificación y uso de recursos, ante los cuales el docente debe responder según la frecuencia con que las realiza eligiendo entre cinco alternativas (Nunca, Casi nunca, A veces, Casi siempre y Siempre). El cuestionario fue elaborado por 
Pérez et al., que en un estudio psicométrico en docentes chilenos identificó seis factores para el instrumento: Enseñanza centrada en el estudiante, Planificación de la enseñanza, Evaluación de proceso, Relación dialogante, Enseñanza centrada en el profesor y Uso de recursos tecnológicos ${ }^{12}$.

- Inventario de estrategias de aprendizaje de $R$. Schmeck: Instrumento para la detección y medición de las estrategias de aprendizaje, que presenta 55 ítems con comportamientos académicos ante los cuales el participante debe responder verdadero o falso. Evalúa cuatro factores: Pensamiento elaborativo, Estudio metódico, Pensamiento profundo y Retención de hechos. El cuestionario ha mostrado evidencia de su fiabilidad, validez predictiva $y$ de constructo ${ }^{13}$.

\section{Procedimiento}

Los docentes fueron contactados individualmente, y la aplicación de los cuestionarios se realizó en un lugar y momento acordado previamente con ellos. La aplicación fue realizada por la investigadora, luego de realizar un proceso de consentimiento informado visado por el Comité de Bioética de la Facultad de Medicina de la Universidad de Concepción.

\section{Análisis de datos}

Se realizó un análisis descriptivo de ambos cuestionarios, evaluando su confiabilidad aplicando alfa de Cronbach y sus estadísticos de tendencia central y dispersión. La correlación entre prácticas pedagógicas y estrategias de aprendizaje se evaluó con el coeficiente de Pearson según contraste unilateral, y la capacidad predictiva conjunta de las estrategias de aprendizaje sobre las prácticas pedagógicas se hizo mediante regresión lineal múltiple. Se consideró un $\mathrm{p}<0,05$ como estadísticamente significativo. El análisis estadístico se realizó en STATA SE 11.0.

\section{Resultados}

Considerando los rangos habituales de interpretación del alfa de Cronbach (mayor o igual a 0,9: Excelente; 0,8 a 0,9: Muy buena; 0,7 a 0,8: Buena; 0,6 a 0,7: Aceptable; 0,5 a 0,6: Pobre; menor a 0,5 : Inaceptable $)^{14}$, se encontró que ambos cuestionarios exhibían una medición suficientemente precisa para usarse en investigación (Tablas 1 y 2).

Tabla 1. Estadísticos descriptivos del Cuestionario de Prácticas Pedagógicas en docentes de carreras de la salud

\begin{tabular}{|lcccccc|}
\hline & $\boldsymbol{\alpha}$ & M & DE & Mín & Máx & \% \\
\hline Enseñanza centrada en el estudiante & $0,83^{a}$ & 41,26 & 5,11 & 24 & 50 & 78,14 \\
\hline Planificación de la enseñanza & 0,87 & 47,49 & 7,60 & 20 & 60 & 73,94 \\
\hline Evaluación de proceso & 0,81 & 34,49 & 7,09 & 13 & 49 & 61,21 \\
Relación dialogante & 0,79 & 35,31 & 3,64 & 22 & 40 & 85,34 \\
\hline Enseñanza centrada en el profesor & 0,67 & 20,36 & 4,22 & 8 & 28 & 59,83 \\
Uso de recursos tecnológicos & 0,57 & 18,97 & 3,05 & 10 & 25 & 69,83 \\
\hline
\end{tabular}

$\mathrm{N}=200 ; \alpha=$ Alfa de Cronbach; $M=$ Media aritmética; DE = Desviación estándar; Mín = Mínimo; Máx = Máximo; \% = (Puntaje observado-puntaje mínimo posible)/(puntaje máximo posible-puntaje mínimo posible)¹00.

Tabla 2. Estadísticos descriptivos del Inventario Schmeck de estrategias de aprendizaje en docentes de carreras de la salud

\begin{tabular}{|lcccccc|}
\hline & $\boldsymbol{\alpha}$ & M & DE & Mín & Máx & \% \\
\hline Pensamiento elaborativo & 0,63 & 6,80 & 1,50 & 2 & 8 & 85,07 \\
Estudio metódico & 0,66 & 10,27 & 2,80 & 4 & 20 & 68,64 \\
\hline Pensamiento profundo & 0,54 & 13,67 & 2,92 & 2 & 36 & 85,65 \\
Retención de hechos & 0,57 & 4,04 & 2,43 & 0 & 13 & 25,24 \\
\hline
\end{tabular}

$\mathrm{N}=200 ; \alpha=$ Alfa de Cronbach; $M=$ Media aritmética; DE = Desviación estándar; Mín = Mínimo; Máx = Máximo; \% = (Puntaje observado-puntaje mínimo posible)/(puntaje máximo posible-puntaje mínimo posible)¹00. 
Dada la ausencia de baremos locales para ambos instrumentos, se procedió a calcular un porcentaje de logro (\%) de acuerdo al puntaje máximo de ellos. Aunque no constituyen baremos para clasificar sus puntuaciones, son una alternativa orientadora para interpretarlos.

Así, en el CPP, se identificó un mayor nivel de relación dialogante $(85,34 \%)$ y un menor nivel de enseñanza centrada en el profesor $(59,83 \%)$ en los encuestados (Figura 1). En el Schmeck, se encontró que los docentes presentaban mayor pensamiento elaborativo $(85,07 \%)$ y pensamiento profundo $(85,65 \%)$, con un mucho menor uso de estrategias de retención de hechos $(25,24 \%)$ (Figura 2).

Al correlacionar las prácticas pedagógicas y estrategias de aprendizaje en los docentes, se encontró que un mayor uso de estrategia de pensamiento elaborativo se asociaba, de manera estadísticamente significativa, con una enseñanza más centrada en el estudiante $(\mathrm{p}<0,001)$, mayor

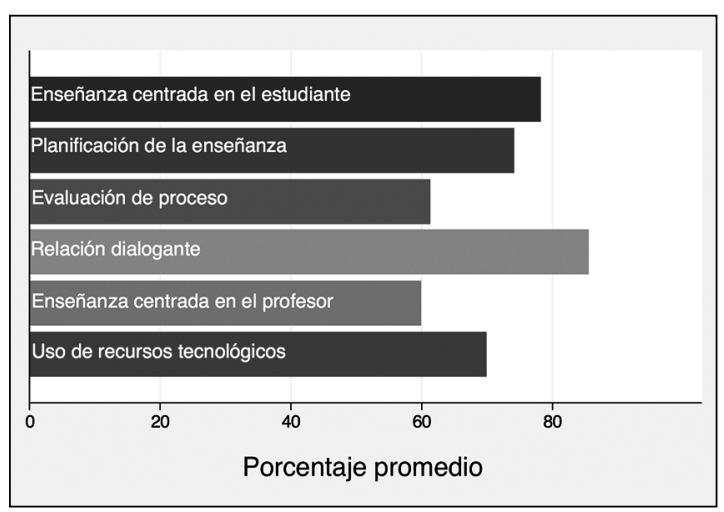

Figura 1. Puntuaciones porcentuales promedio de los factores del Cuestionario de Prácticas Pedagógicas en docentes de la salud. planificación de la enseñanza $(\mathrm{p}<0,001)$, mayor evaluación de proceso $(\mathrm{p}<0,05)$ y mayor relación dialogante $(\mathrm{p}<0,05)$.

En tanto, un mayor nivel de estudio metódico se asoció a una enseñanza más centrada en el estudiante $(\mathrm{p}<0,05)$, mayor planificación de la enseñanza $(\mathrm{p}<0,001)$, mayor evaluación de proceso $(\mathrm{p}<0,05)$, mayor relación dialogante $(\mathrm{p}<0,05)$ $y$ mayor uso de recursos tecnológicos $(p<0,05)$.

El pensamiento profundo mostró correlaciones similares con la enseñanza centrada en el estudiante $(\mathrm{p}<0,01)$, la planificación de la enseñanza $(\mathrm{p}<0,001)$, la evaluación de proceso $(\mathrm{p}<0,001)$, la relación dialogante $(\mathrm{p}<0,001), \mathrm{y}$ el uso de recursos tecnológicos $(\mathrm{p}<0,05)$.

De manera opuesta, una mayor retención de hechos se asoció a una enseñanza menos centrada en el estudiante $(\mathrm{p}<0,001)$, menor planificación de la enseñanza $(\mathrm{p}<0,01)$ y menor evaluación de proceso $(\mathrm{p}<0,01)$ (Tabla 3$)$.

Posteriormente, para identificar el efecto con-

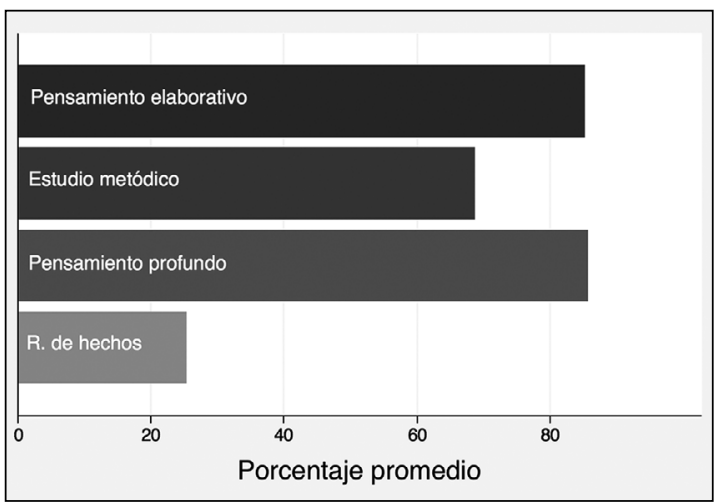

Figura 2. Puntuaciones porcentuales promedio de los factores del Inventario de estrategias de aprendizaje de Schmeck en docentes de la salud.

Tabla 3. Correlación entre las prácticas pedagógicas y las estrategias de aprendizaje en docentes de carreras de la salud

\begin{tabular}{|lcccc|}
\hline & $\begin{array}{c}\text { Pensamiento } \\
\text { elaborativo }\end{array}$ & $\begin{array}{c}\text { Estudio } \\
\text { metódico }\end{array}$ & $\begin{array}{c}\text { Pensamiento } \\
\text { profundo }\end{array}$ & $\begin{array}{c}\text { Retención de } \\
\text { hechos }\end{array}$ \\
\hline Enseñanza centrada en el estudiante & $0,24^{* * *}$ & $0,18^{*}$ & $0,27^{* * *}$ & $-0,23^{* *}$ \\
\hline Planificación de la enseñanza & $0,25^{* * *}$ & $0,24^{* * *}$ & $0,21^{* *}$ & $-0,23^{* *}$ \\
\hline Evaluación de proceso & $0,18^{*}$ & $0,15^{*}$ & $0,23^{* *}$ & $-0,23^{* *}$ \\
\hline Relación dialogante & $0,17^{*}$ & $0,18^{*}$ & $0,23^{* * *}$ & $-0,12$ \\
\hline Enseñanza centrada en el profesor & 0,11 & 0,07 & $-0,02$ & 0,06 \\
\hline Uso de recursos tecnológicos & 0,10 & $0,15^{*}$ & $0,18^{*}$ & $-0,13$ \\
\hline
\end{tabular}

$N=200 ;$ a Coeficiente de Correlación de Pearson; * $p<0,05 ; * *: p<0,01 ; * * *: p<0,001$. 
junto de las cuatro estrategias de aprendizaje sobre las prácticas pedagógicas, se realizó un análisis de regresión lineal múltiple considerando cada factor de las prácticas pedagógicas como variable dependiente, y las estrategias de aprendizaje como variables independientes (Tabla 4).

En el caso del primer factor, las estrategias de aprendizaje en su conjunto hicieron una predic- ción estadísticamente significativa de $13,14 \%$ de este factor $(\mathrm{p}<0,001)$. Considerando cada predictor individualmente, un mayor Pensamiento elaborativo $(\mathrm{p}<0,05)$ y un mayor Pensamiento profundo $(\mathrm{p}<0,01)$, realizaron una predicción significativa de una enseñanza más centrada en el estudiante, explicando $2,91 \%$ y $3,98 \%$ de esta forma de enseñanza, respectivamente.

Tabla 4. Resultados de la regresión lineal múltiple del efecto de las estrategias de aprendizaje sobre los seis factores de prácticas pedagógicas

\begin{tabular}{|c|c|c|c|c|c|}
\hline Variable dependiente & Predictores & B & EE & $\boldsymbol{\beta}$ & $s \mathbf{r}^{2}$ \\
\hline $\begin{array}{l}\text { Enseñanza centrada en el } \\
\text { estudiante }\end{array}$ & $\begin{array}{l}\text { Constante } \\
\text { Pensamiento elaborativo } \\
\text { Estudio metódico } \\
\text { Pensamiento profundo } \\
\text { Retención de hechos } \\
\mathrm{R}^{2}=0,15^{* * *} ; \mathrm{R}^{2} \text { ajustado }=0,13\end{array}$ & $\begin{array}{c}30,91 \\
0,60^{*} \\
0,22 \\
0,37^{* *} \\
-0,26\end{array}$ & $\begin{array}{l}0,24 \\
0,12 \\
0,12 \\
0,15\end{array}$ & $\begin{array}{r}0,18 \\
0,12 \\
0,21 \\
-0,12\end{array}$ & $\begin{array}{l}0,03 \\
0,01 \\
0,04 \\
0,01\end{array}$ \\
\hline Planificación de la enseñanza & $\begin{array}{l}\text { Constante } \\
\text { Pensamiento elaborativo } \\
\text { Estudio metódico } \\
\text { Pensamiento profundo } \\
\text { Retención de hechos } \\
R^{2}=0,15^{* * *} ; R^{2} \text { ajustado }=0,14\end{array}$ & $\begin{array}{c}32,61 \\
0,93^{* *} \\
0,54^{* *} \\
0,35 \\
-0,42\end{array}$ & $\begin{array}{l}0,35 \\
0,18 \\
0,18 \\
0,22\end{array}$ & $\begin{array}{r}0,18 \\
0,20 \\
0,13 \\
-0,13\end{array}$ & $\begin{array}{l}0,03 \\
0,04 \\
0,02 \\
0,02\end{array}$ \\
\hline Evaluación de proceso & $\begin{array}{l}\text { Constante } \\
\text { Pensamiento elaborativo } \\
\text { Estudio metódico } \\
\text { Pensamiento profundo } \\
\text { Retención de hechos } \\
\mathrm{R}^{2}=0,11^{* * *} ; \mathrm{R}^{2} \text { ajustado }=0,09\end{array}$ & $\begin{array}{c}24,47 \\
0,55 \\
0,26 \\
0,39^{*} \\
-0,44^{*}\end{array}$ & $\begin{array}{l}0,33 \\
0,17 \\
0,17 \\
0,21\end{array}$ & $\begin{array}{r}0,12 \\
0,10 \\
0,16 \\
-0,15\end{array}$ & $\begin{array}{l}0,01 \\
0,01 \\
0,02 \\
0,02\end{array}$ \\
\hline Relación dialogante & $\begin{array}{l}\text { Constante } \\
\text { Pensamiento elaborativo } \\
\text { Estudio metódico } \\
\text { Pensamiento profundo } \\
\text { Retención de hechos } \\
\mathrm{R}^{2}=0,10^{* * *} ; \mathrm{R}^{2} \text { ajustado }=0,08\end{array}$ & $\begin{array}{c}28,10 \\
0,34^{*} \\
0,17 \\
0,24^{*} \\
-0,04\end{array}$ & $\begin{array}{l}0,17 \\
0,09 \\
0,09 \\
0,11\end{array}$ & $\begin{array}{r}0,14 \\
0,13 \\
0,20 \\
-0,03\end{array}$ & $\begin{array}{r}0,02 \\
0,02 \\
0,03 \\
<0,01\end{array}$ \\
\hline $\begin{array}{l}\text { Enseñanza centrada en el } \\
\text { profesor }\end{array}$ & $\begin{array}{l}\text { Constante } \\
\text { Pensamiento elaborativo } \\
\text { Estudio metódico } \\
\text { Pensamiento profundo } \\
\text { Retención de hechos } \\
\mathrm{R}^{2}=0,03 ; \mathrm{R}^{2} \text { ajustado }=0,01\end{array}$ & $\begin{array}{r}16,36 \\
0,38 \\
0,09 \\
-0,01 \\
0,17\end{array}$ & $\begin{array}{l}0,21 \\
0,11 \\
0,11 \\
0,13\end{array}$ & $\begin{array}{r}0,13 \\
0,06 \\
-0,01 \\
0,10\end{array}$ & $\begin{array}{r}0,02 \\
<0,01 \\
<0,01 \\
0,01\end{array}$ \\
\hline Uso de recursos tecnológicos & $\begin{array}{l}\text { Constante } \\
\text { Pensamiento elaborativo } \\
\text { Estudio metódico } \\
\text { Pensamiento profundo } \\
\text { Retención de hechos } \\
\mathrm{R}^{2}=0,06^{*} ; \mathrm{R}^{2} \text { ajustado }=0,04\end{array}$ & $\begin{array}{r}15,29 \\
0,13 \\
0,13 \\
0,14 \\
-0,10\end{array}$ & $\begin{array}{l}0,15 \\
0,08 \\
0,08 \\
0,09\end{array}$ & $\begin{array}{r}0,06 \\
0,12 \\
0,13 \\
-0,08\end{array}$ & $\begin{array}{r}<0,01 \\
0,01 \\
0,02 \\
0,02\end{array}$ \\
\hline
\end{tabular}

$B=$ coeficientes de regresión no estandarizados; $E E=$ errores estándar; $\beta=$ coeficientes de regresión estandarizados; $\mathrm{sr}^{2}=$ correlaciones semiparciales al cuadrado; $\mathrm{R}^{2}=$ coeficiente de determinación; $\mathrm{R}^{2}=$ coeficiente de determinación ajustado. $\mathrm{N}=200 ;{ }^{*} \mathrm{p}<0,05 ;{ }^{* *} \mathrm{p}<0,01 ;{ }^{* *} \mathrm{p}<0,001$. 
Para el segundo factor, las estrategias de aprendizaje juntas hicieron una predicción estadísticamente significativa de $13,52 \%$ de este factor $(\mathrm{p}<0,01)$. Individualmente, un mayor Pensamiento elaborativo $(\mathrm{p}<0,01)$ y un mayor Estudio metódico $(\mathrm{p}<0,01)$, se asociaron a una planificación más frecuente, explicando 3,07\% y $3,77 \%$ de ésta respectivamente.

En cuanto a evaluación de procesos, las estrategias de aprendizaje predijeron $8,93 \%$ de ésta $(\mathrm{p}<0,001)$. Individualmente, un mayor Pensamiento profundo $(\mathrm{p}<0,01)$, y un menor Estudio metódico $(\mathrm{p}<0,01)$, realizaron una predicción significativa de una evaluación de proceso más frecuente, dando cuenta de $2,37 \%$ y $1,98 \%$ de su variación, respectivamente.

En cuanto al factor de relación dialogante, las cuatro estrategias de aprendizaje hicieron una predicción estadísticamente significativa de 7,75\% de éste $(\mathrm{p}<0,001)$. Individualmente, un mayor Pensamiento elaborativo $(\mathrm{p}<0,05)$ y un mayor Pensamiento profundo ( $\mathrm{p}<0,01)$, se asociaron significativamente a una mayor frecuencia de actividades dirigidas a desarrollar esta relación, dando cuenta de $1,82 \%$ y $3,47 \%$ de su variación, respectivamente.

Para el factor de enseñanza centrada en el profesor, las cuatro estrategias de aprendizaje no hicieron una predicción estadísticamente significativa de éste $(\mathrm{p}=0,27)$.

Finalmente, en cuanto al factor de uso de recursos tecnológicos, las estrategias de aprendizaje predijeron significativamente $3,81 \%$ de éste $(\mathrm{p}<0,05)$. Pero ningún predictor mostró una capacidad predictiva significativa individualmente.

\section{Discusión}

Se observó un uso frecuente de distintos tipos de prácticas pedagógicas, tanto constructivistas como tradicionales, mostrando casi todas una alta frecuencia comparadas con el estudio de Pérez ${ }^{12}$, excepto el uso de recursos tecnológicos que mostró una frecuencia promedio.

Esto puede deberse a que dos tercios de los participantes contaban con estudios de postgrado (un tercio, en temáticas educativas). Un estudio chileno encontró que sólo tener docentes con postgrado disminuye los niveles de deserción de los estudiantes y mejora su desempeño ${ }^{15}$. Esto, pues un cuerpo académico capacitado y con dominio especializado de su disciplina, mejoraría la docencia y favorecería la retención del alumnado, ayudando a que pueda concluir exitosamente sus estudios $^{16-18}$. Además, docentes más capacitados tenderían a una docencia centrada en el estudiante ${ }^{18,19}$.

Ahora, que el uso de recursos tecnológicos presente un nivel medio puede deberse a que la práctica docente es un fenómeno multi-determinado ${ }^{9}$. Por ello, el uso de recursos tecnológicos requiere también actitudes favorables y capacitación previa en tecnología para los docentes, factores no abordados en este estudio. Asimismo, los postgrados no necesariamente consideran el uso de recursos tecnológicos, y la capacitación en estas herramientas puede ser un factor mediador para su uso ${ }^{20}$.

También se encontró que los docentes realizan prácticas de enseñanza tanto centradas en el profesor como centradas en el estudiante, lo que puede deberse a que las primeras aún son predominantes en el sistema educacional chileno, y pudieron parecerle útiles a los docentes cuando fueron estudiantes ${ }^{18,19}$. Sin embargo, paralelamente, cambios en educación médica, el desarrollo del constructivismo y el interés de las instituciones en capacitar a sus docentes, habría aumentado las prácticas de enseñanza centrada en el estudiante ${ }^{21}$.

Ante esto, es necesario mencionar que no necesariamente es negativo utilizar metodologías tradicionales. Por el contrario, es deseable utilizar diversas metodologías docentes, aplicando la más apropiada a los objetivos perseguidos ${ }^{17,21}$.

En cuanto a las estrategias de aprendizaje de los docentes, los participantes mostraron una mayor frecuencia de estrategias de aprendizaje más complejas, profundas y elaborativas ${ }^{11}$.

Schön sostiene que cada vez que se utiliza un conocimiento, éste se reaprende, se contextualiza y se incrementa el saber generando así niveles de aprendizaje más profundos ${ }^{22}$. Esto podría implicar que los docentes del estudio, desarrollan estrategias de aprendizaje más complejas pues son éstas las que les permiten alcanzar un mayor nivel de experticia para desenvolverse en las distintas dimensiones del rol docente ${ }^{23}$.

En contraposición, los docentes utilizan en menor medida estrategias de aprendizaje mecánico, como la retención de hechos ${ }^{11,24}$, presentando un escenario altamente deseable para la formación 
universitaria, ya que las estrategias mecánicas dificultarían más que los docentes alcanzaran la experticia necesaria para formar a los estudiantes ${ }^{9,11}$.

En cuanto a las correlaciones, se encontró que existe relación entre prácticas y estrategias de aprendizaje de los docentes, lo que coincide con la literatura ${ }^{18,19}$. Los docentes que aprenden mediante retención de hechos tienen menos prácticas constructivistas. Dado que las actividades constructivistas favorecen el aprendizaje profundo, es esperable que quienes no tienden a recurrir a este tipo de aprendizaje, eventualmente no vean la necesidad de favorecerlo en sus estudiantes ${ }^{16}$. Es posible que estos docentes prefieran prácticas expositivas, que de usarse en exclusiva, serían las menos beneficiosas para la formación ${ }^{16,25}$.

Por otro lado, los docentes que tienden al procesamiento profundo y elaborativo mostraron preferencia por las prácticas constructivistas, que justamente favorecen el tipo de aprendizaje que ellos mismos presentan ${ }^{16}$. Estas prácticas, además de ser las que promueve la Educación Médica, estarían asociadas a un mejor rendimiento académico en los estudiantes ${ }^{11}$.

En los resultados de la regresión lineal se observa que las estrategias de aprendizaje están asociadas a la forma en que el docente enseña, explicando hasta $15 \%$ de las prácticas pedagógicas. Esto implica que hay al menos $85 \%$ de la variación de estas prácticas que pueden estar asociadas a otros factores, pudiendo ser tanto individuales como ambientales ${ }^{12,18,25}$.

Sin embargo, sí se observa que el pensamiento profundo es el principal predictor, junto con el pensamiento elaborativo, de la enseñanza centrada en el estudiante y la relación dialogante, favoreciendo ambas. Asimismo, promueve la evaluación de proceso.

Lo anterior evidenciaría que la forma de aprender del docente estaría asociada a cómo dicta su docencia.

Sin embargo, la enseñanza centrada en el profesor y el uso de recursos tecnológicos no se asociarían a las estrategias de aprendizaje, lo que podría deberse a que la primera constituye, como ya se indicó, el estándar remanente de la enseñanza chilena y a que el uso de tecnología demanda otros factores coadyuvantes, como la capacitación ${ }^{4,26}$.

Queda, para futuras investigaciones, abordar la relación de las prácticas y estrategias de aprendi- zaje docentes con características socioecónomicas y laborales de los académicos, así como con los resultados del estudiantado.

\section{Referencias}

1. Román M, Diéz E. El Currículum como desarrollo de procesos cognitivos y afectivos. Revista Enfoques Educacionales 2000; 2: 1-17.

2. Malagón L. El currículo: dispositivo pedagógico para la vinculación Universidad-Sociedad. Revista ieRed 2004; 1: 1-28.

3. Moya M, Hernández J, Hernández JR, Cózar R. Un estilo de aprendizaje, una actividad. Diseño de un plan de trabajo para cada estilo. Revista Estilos de Aprendizaje 2009; 2: 140-52.

4. Jones R, Higgs R, de Angelis C, Prideaux D. Changing face of medical curricula. Lancet 2001; 357: 699-703.

5. Ortiz L. Editorial. Rev Educ Cienc Salud 2010; 7: 5-6.

6. Ibáñez P, Fasce E, Ramírez L. Modernización de la enseñanza en la Medicina: el aprendizaje basado en problemas. Universidad de Concepción, 1996. Concepción.

7. Castilla M, López C. Los roles del docente en la educación médica. EducEduc 2007; 10: 105-13.

8. Cortez K, Fuentes V, Villablanca I, Guzmán C. Creencias docentes de profesores ejemplares y su incidencia en las prácticas pedagógicas. Estud pedagóg 2013; 39 (2): 97113.

9. Contreras J. La práctica docente y sus dimensiones. Valoras UC 2003; 1-3. Disponible en: http://valoras.uc.cl. [Consultado el 10 de julio de 2014].

10. Valcárcel Pérez MS, Verdú MJ. Observación y evaluación de la enseñanza comunicativa de lenguas modernas. Madrid, 1996. Ministerio de Educación y Ciencia.

11. Fernández $\mathrm{O}$, Martínez-Conde M, Melipillán R. Estrategias de aprendizaje y autoestima. Su relación con la permanencia y deserción universitaria. Estud pedagóg 2009; 35: 27-45.

12. Pérez C, Vaccarezza G, Aguilar C, Coloma K, Salgado $\mathrm{H}$, Baquedano $\mathrm{M}$, et al. Cuestionario de prácticas pedagógicas: Análisis de su estructura factorial y consistencia interna en docentes de carreras de la salud. Rev Med Chile (en revisión).

13. Schmeck R. Inventory of Learning Processes. En: Students Learning Styles and Brain Behavior. ERIC Editors. Ann Arbor, Michigan, 1982.

14. George D, Mallery P. SPSS for Windows step by step: A simple guide and reference. 11.0 update (4th ed.). Boston: Allyn \& Bacon; 2003.

15. Araneda-Guirriman C, Rodríguez-Ponce E, Pedraja- 
Rejas L. Relación entre el financiamiento fiscal, la calidad del cuerpo académico y la retención de estudiantes universitarios en Chile. Formación universitaria 2013; 6: 55-64.

16. Fasce E, Ibáñez P. Fundamentos y práctica de la educación médica. Concepción: Universidad de Concepción, 2013.

17. Santibáñez M. Desafíos educativos para el profesor-médico. Reflexiones desde la educación. ARS Médica (s/f). Disponible en: http://escuela.med.puc.cl/publ/arsmedica/ArsMedica.html. [Consultado el 20 de julio 2015].

18. Brown BL. Teaching Style vs Learning Style. Myths and Realities. ERIC 2003; 26: 1-3.

19. Gilakjani AP. A match or mismatch between learning styles of the learners and teaching styles of the teachers. IJMECS 2012; 4 (11): 51-60.

20. Kunakov N. Escuelas de Medicina: los estudiantes de hoy. Rev Med Chile 2011; 139: 524-8.

21. Coppola B. The Distinctiveness of Higher Education. Journal of Chemical Education 2013; 90: 955-6.
22. Schön D. La formación de profesionales reflexivos: hacia un nuevo diseño de la enseñanza y el aprendizaje en las profesiones. Barcelona: Paidós, 1992.

23. López A, González I, De León C. Perfil de un buen docente. Aplicación de un protocolo de evaluación de las competencias del profesorado universitario. Revista Electrónica Interuniversitaria de Formación del Profesorado 2014; 17: 133-48.

24. Acevedo C, Chiang T, Madrid H, Reinicke F. Estrategias de aprendizaje en alumnos universitarios y de enseñanza media. Revista Estilos de Aprendizaje 2009; 2: 114-26.

25. Feixas M. Enfoques y concepciones docentes en la Universidad. Revista Electrónica de Investigación y Evaluación Educativa 2010; 16: 1-27.

26. López de la Madrid M, Espinoza A, Flores K. Percepción sobre las tecnologías de la información y la comunicación en los docentes de una universidad mexicana: el Centro Universitario del Sur de la Universidad de Guadalajara. Revista Electrónica de Investigación Educativa 2006; 8: 1-14. 